\title{
From health behaviours to health practices: an introduction Simon Cohn
}

\author{
Abstract
}

The concept of health behaviour has become ubiquitous in health-related research and intervention studies, as well as among policymakers. Developed from psychology, it is based on a number of key underlying assumptions that enable it to be integrated in an existing health research paradigm. However, by conceiving individual health behaviour as discrete, stable, homogeneous and measurable, many other aspects of health-related activities, in particular those relating to power and sociality, are excluded. As a consequence, any genuine contribution from medical sociology or related disciplines is, at best, limited. To counter this, it is proposed that reconceptualising what people do in terms of health practices, rather than health behaviour, captures the emergent and contingent properties of people's activities in particular situations. Rather than serving as a direct replacement term, and thus reproducing the same epistemological assumptions, it is argued that its very flexibility and capacity to articulate different theoretical orientations is likely to be its major strength.

Changes in demographics and the widespread decline of communicable disease in both Western and non-Western populations has resulted in the rise of so-called chronic illnesses, and in particular, 'lifestyle conditions'. This has led health professionals to turn their gaze from eradicating external agents of disease to altering a wide range of interacting factors identified as causing, or having the potential to cause, ill health. As a result, the idea that smoking, diet, alcohol consumption and physical activity should now be a central focus for intervention has become an established and uncontested position not only in health research but among policymakers, the media and the public more generally.

Derived from health psychology, the concept of health behaviour underpins many of these developments and has led to the generation of new typologies and fields of academic expertise. The assumption that there are easily identifiable and observable forms of health behaviour has almost universally been adopted by those involved in (and funded to do) healthcare research. Yet a great wave of research over the last two decades attempting to develop techniques and evidence of behavioural change has proved to have surprisingly limited success. Usually, explanations of negative findings focus on the nature of the intervention, its theoretical underpinnings, problems with its delivery or the outcome measures used. Very rarely, if ever, is the validity or usefulness of the pivotal concept of health behaviour itself ever questioned. In addition, critics have suggested that the concept insidiously supports variations of neoliberal ideology and responsibilisation, for example through such creative terms as liberal paternalism.

The collection of articles in this collection responds to the fact that surprisingly little critical attention has been paid to how health behaviour is actually conceptualised, whether this might explain why attempts to change what people do for any extended period seem to be so difficult, and what the limitations of the term might be. Collectively, these contributions show that across this burgeoning corpus of work there is remarkably little discussion of power or conceptualisation of sociality beyond a largely epidemiological idea of population. They explore whether there are alternative ways to both theorise and conduct research into what people do and don't do in relation to their health. And, given that very little in this field of research has attempted to capture the specific and detailed qualities of people's activities in particular situations, they demonstrate how investigating local minutiae might actually be crucial to provide general insight. Thus, by drawing on a range of theoretical approaches and empirical studies, the articles highlight the potential of 
sociology, and the social sciences more generally, to provide complementary or alternative ways of considering human activities that relate to health.

As a way of introducing the articles, and pre-empting the ways in which they critically engage with the concept of health behaviour, I want to spend just a little time describing something of the underlying logic that determines not simply how health behaviour is conceived in the abstract, but perhaps more significantly how it is constructed through research activities designed to address contemporary health problems. Implicitly I draw on my experiences working in a multidisciplinary team consisting of health psychologists and behavioural scientists, primary and secondary care medics, epidemiologists, statisticians and the like. While this is in part a caricature, my intention is to be provocative. It is not simply to pave the way for the more substantive contributions, but to help catalyse a further debate that needs to be had: that, as social scientists often working alongside other disciplines in health research environments, we have, ourselves, in many instances accepted the concept of health behaviour far too readily.

Originally healthy behaviour (Kasl and Cobb 1966), or health-protective behaviour (Harris and Guten 1979) referred to the strategies people might adopt to prevent disease. In a much cited piece of research, Belloc and Breslow (1972) argued that personal lifestyle (sleep, diet, physical activity and smoking) impacted on health in diffuse ways and that there was a direct correlation between them and the risks of ill-health. Interestingly, Matarazzo (1983) later drew directly on a biomedical paradigm to describe this kind of behaviour as a behavioural pathogen and contrasted this with health-protective behaviour, which he labelled a behavioural immunogen. What was significant was that, by adopting the clear distinction informed by biomedicine that some behaviour is unequivocally good whilst other kinds are detrimental, the behaviour itself became abstract and removed from any comprehensive, detailed description of what people were actually doing. And, although the original emphasis of health behaviour was on its positive role in illness prevention, because the primary focus came to be on behavioural change most research has come to focus on negative health behaviour. Even physical activity has, in recent years, been recast to fit this schema, as research has shifted from its promotion to addressing the 'problem' of sedentarism.

Initial attempts to encourage certain kinds of health behaviour, while discouraging others, were aimed at modifying people's health beliefs through education initiatives. By assuming that what people did was the result of deliberation and reasoning, addressing motives and intentions became central. As a result, Levanthal's self-regulatory model (Leventhal et al. 2003) was highly influential, since it proposed the ways in which individuals reflect on what they do prior to action. Drawing on both individual attitudes and socially determined norms, this psychological approach created the space into which researchers felt confident they could intervene. However, as studies began to report that there is often a gap between intentions and behaviour, Bandura's notion of self-efficacy (1977) was increasingly adopted. Further adapted by Ajzen, this general orientation proposed that as well as attitudes and subjective norms influencing intentions, certain beliefs affect levels of perceived control and that this might explain why intentions are not always translated into actions (Ajzen, 1991). More recently, some psychologists have drawn from behavioural economics to explore alternative ways in which non-deliberate or automatic processes might explain why people behave the way that they do, rather than the more reason-based models that initially shaped behavioural science (Thaler and Sustein, 2008).

A key point in all this, however, is that despite the evolution of the concept of health behaviour in the psychological literature, what has remained central is the linear order that conceives of various psychological determinants, potentially modified by social norms and triggered by environment cues, which then determine someone's behaviour. In other words, health behaviour is seen as the an outcome of an individual who is presented as the obvious focus of both the processes preceding behaviour and the agent of the behaviour itself. Thus, although contemporary behavioural science might not equate directly with classic behaviourism, because of its focus on mediating cognitive processes, behaviour is nevertheless conceived of as a definitive and 
observable entity that should not require abstract concepts or assumptions to identify or interpret it (Baumeister et al. 2007), just as Watson argued a century ago (Watson 1930).

In contemporary research, specific forms of health behaviour tend to be combined with other disease-related elements so that useful, multifactorial accounts can be established (Abraham et al. 2000). They have to be drawn out from the inherently chaotic variation of human activity and sufficiently standardised to resemble other items, such as demographic and physiological factors. Often a range of graphic representations during the research process and in final publications further confirm the epistemological parity between health behaviour and other variables; flow charts, tree diagrams, drawings of multiple boxes and arrows all map out the mix and the hypothesised relationships between them. Two key assumptions underlie this process: that kinds of behaviour can be considered to be distinct from each other and that they can potentially be controlled or altered once an accurate causal explanation is established.

The imperative to consider specific forms of behaviour as unique variables that can be studied and assembled alongside others means that they must be conceived of as discrete, stable, homogeneous, observable and, crucially, measurable. Often a distinction is made between subjective and objective measures. The former consist of various methods of participant selfreporting - usually by questionnaires or diaries - and tend to be regarded as unreliable. In contrast, the term objective measures increasingly refers to various forms of electronic technologies that can record data relatively unobtrusively and independently of the participant. Thus, embedded in the very objects of study is a preconceived notion not only of what they are, but also how they function.

But because research is only conducted on kinds of behaviour that already fulfil these criteria, other health-related activities or variations of what people do in different situations that escape the parameters of measurement are excluded. The social, affective, material and inter-relational features of human activity are effectively eliminated, as behaviour becomes viewed as an outcomes of the individual and determined only by such things as motives, intentions and the subjective reception of norms and cues. In response, there has been a growing acknowledgement that the specifics of context are significant and somehow need to be taken in to account. However, citing context to situate individuals in their physical and social environment indirectly serves to reinforce what is of primary importance and fore grounded. Health behaviour remains contrasted against a backdrop of interrelated factors that fall outside the specifics of research because they have not, or often cannot, be rendered into variables. As a consequence, although discussion of context may ostensibly resemble adoption of a more sociological perspective, by preserving the delineated characteristics of health behaviour and pre-empting a focus for causal explanation, its inclusion frequently serves simply to maintain, rather than revise, conceptualisations of health behaviour.

So why is problematising the category of health behaviour important? After all, it would be reasonable to argue that not only are these weaknesses inevitable, but they are actually highly productive and necessary in order to construct a particular kind of object of enquiry. But a danger of this is that the notion of behaviour becomes so reified that it fails to provide any critical insight into what people actually do and why. In this, then, lies the inherent conservatism of adopting categories of behaviour a priori. The issue is not simply that they continuously get reproduced from one research project to another but that they increasingly become naturalised the more they are 'understood'. It is also clear that the focus on health behaviour unavoidably presents a particular moral explanation, as issues of responsibility and agency are distributed in specific ways along causal pathways that inevitably converge on the individual. Although not necessarily intentional, this individualising characteristic can all too easily align itself, and further legitimate, public health and policy strategies that ignore the complex structural issues that underpin the political economy of health. 
This collection of articles contends, in different ways, that many of the limitations and consequences of the concept of health behaviour can be potentially addressed by drawing on a broader notion of health practices. In contrast to the idea of specific behaviour, everyday practices are always locally situated and composite. They are not a direct result or outcome of mental processes but emerge out of the actions and interactions of individuals in a specific context. Thus, the word practice has the potential to resist both the psychologising and the individualising features that ultimately have come to define the term health behaviour. However, adopting such a conception means it is perhaps impossible and even undesirable to try and identify when exactly an action starts and when it ends, or the extent to which one action is distinct from another. It also potentially resists the search for causal explanations, in the form of identifying determinants, and instead embraces the idea that practices are contingent on a whole variety of social and material factors. This is not, however, an argument designed to champion the impossibility of the social sciences genuinely engaging with contemporary health problems. But by being aligned with a language of systems, complexity, interactions and irreducibilities, such an approach must inevitably be modest, since it can only ever offer a partial interpretation. Nevertheless, it also means that issues of power and politics cannot be bracketed off but must be recognised as central features of any proposed method to influence or change people.

Identifying a variety of problems associated with the concept of health behaviour, and considering what possibilities a more practice-orientated approach might offer, the contributions in this monograph address many of the issues raised above in a variety of ways. As a result, grouping articles together represents just one way in which the overlapping and cross-cutting themes in the collection might be navigated. A first set explores some of the theoretical approaches from sociology that have the potential to provide a counter to the individualised notion of health behaviour. To begin with, Armstrong argues that the whole shift towards encouraging patients to take control of their behaviour and, as a consequence accept responsibility, is remarkably recent. Importantly, he also suggests that ultimately agency itself has, in the process, come to be equated with health. Johnson and Horricks extend this theme by critically examining the ideological assumptions associated with such things as self-management and choice. They describe the ways in which health psychology has served to support and reproduce a range of values that inevitably engage more favourably with some groups of people more than others. Veenstra and Burnett also tackle the concept of agency through the influential work of Bourdieu and in particular through his rendering of structure-agency as co-constituting rather than in opposition. They argue, however, that the relational characteristics of many of Bourdieu's terms tend to be underemphasised and that, if accounts of health practice are to be of value for applied work, it is crucial to embrace this ontological imperative. Finally, Frohlich and Abel return to the unequal distribution of health and health-resources with the observation that those who are most deprived are the least likely to alter their health related practices. By integrating Sen's capability theory with the theories of Bourdieu, the authors make a shift from framing the issue in terms of who possesses what capital to thinking about where there might be social and cultural opportunities for change.

A second group of articles draw on accounts of particular health initiatives to explore the limitations of behaviour-based interventions and the potential for social science approaches in current health research. In different ways they extend the general theoretical perspectives introduced by the initial articles to illustrate the extent to which people's actions arise from their interactions both with other people and the material environment. Implicitly drawing on arguments of scale, the articles present a variety of cases in which apparently individual actions are influenced by forces that can only be conceived of at a social level. This implicitly alters not merely the focus of causal explanations but highlights the ways in which practices emerge from, and are always contingent upon, a wide range of factors that operate alongside, rather than 'prior to'. As some of the authors explicitly propose, this offers new ways to think about health interventions that need to take into account factors that defy reductionism. Firstly, Baum and 
Fisher continue with the theme of health inequalities and discuss how, despite the weight of evidence for the existence of social determinants of health, governments nevertheless are attracted to behavioural explanations for ideological reasons. Given this trend, they conclude that health policies are unlikely to ever address the broader social foundations of health. Ong et al. take up the same issue through a discussion of context and, using two studies as illustrative examples, argue that what is commonly framed as the background features of living with a chronic condition should be refigured to be integrated in existing research designs. Nettleton and Green then draw on case studies to discuss a number of practices relating to transport and physical activity and suggest that a Bourdieusian-informed approach can demonstrate the extent to which existing tacit knowledge and habitus shape the conditions of possibility for change, and therefore that public health must attend to their significance.

The next three articles extend these arguments by providing detailed qualitative accounts of specific health practices. Addressing the health issue of substance misuse by women who are pregnant, Benoit et al. point out that the key to the effectiveness of any intervention is the way that people see substance abuse as problematic. The authors show that views on such things as health risks should not be regarded as individually held beliefs that go on to directly influence behaviour. Instead, they are inherently social discourses that are only meaningful through the ways in which participants talk, compare and situate themselves in relation to others. Complementing this position, Lyons et al. focus on the alcohol consumption of people in their mid-life. Similarly rejecting any approach that assumes that drinking is the result of rational decision-making, they describe how many of the factors that shape alcohol consumption are inherently cultural and embodied. In particular, they include aspects relating to gender and, although this is frequently omitted in health behaviour research, the desire to be moderately intoxicated. Finally, adopting a more overtly anthropological orientation, van der Sijpt explores issues relating to reproduction and contraception in Cameroon. Like many of the other articles, the author explicitly rejects behavioural models that rely on any version of rational choice theory. But she adds to this critique by arguing the same values underlie the notion of individual rights and that these do necessarily translate easily to other cultural contexts. Instead, van der Sijpt suggests that the idea of navigation better captures the practices by which women engage with the different options on contraception and reproduction that they encounter in their daily lives.

The final pair of articles could be said to critique the concept of health behaviour not by drawing on established perspectives of the social but by conceiving of practice as a means to resist seeking direct causal antecedents - whether they be individual psychological processes or, indeed, more diffuse social influences. Will and Weiner examine people's talk about cholesterol reduction and note the ways that people often accommodate contradictory and inconsistent accounts. Beyond an analysis of discourse, their argument suggests that the processes of talking and making sense of everyday practices never need to be fixed or singular because ultimately these are accounts of activities that are not coherent; they are practices, not behaviour. Finally Vogel and Mol explore how the drive for healthy eating, and the various forms of knowledge that support this, have become the antithesis of eating practices associated with pleasure. They argue that linking eating practices with enjoyment is not natural but a relationship that is acquired. By attending to the variety of social and material elements that enable such practices to be pleasurable, an alternative strategy to traditional interventions intended to correct unhealthy behaviour could potentially be developed.

No overview of the articles, especially as brief as this, could claim they all share common approaches or conclusions. Nevertheless, it is clear all the authors are uneasy about the dominance of the concept of health behaviour, based on theoretical, methodological or political grounds. The articles suggest that the concept is too contained, too delineated and too far removed from everyday social life. So perhaps this, then, is the final value of reading the articles together - that 
the term health practices ultimately is useful not because it claims to be a direct alternative, or substitute, for psychological and behavioural approaches but precisely because it allows for sufficient degrees of freedom such that no single theory can ever fully stabilise how it can or should be applied.

\section{References}

Abraham, C., Norman, P. and Conner, M. (eds) (2000) Understanding and Changing Health Behaviour: From Health Beliefs to Self-regulation. Amsterdam: Harwood Academic Press.

Ajzen, I. (1991) The theory of planned behaviour, Organizational Behavior and Human Decision Processes, $50,2,179-211$.

Bandura, A. (1977) Self-efficacy: toward a unifying theory of behavior change, Psychological Review, 84, 2, 191-215.

Baumeister, R., Vohs, K. and Funder, D. (2007) Psychology as the science of self-reports and finger movements: whatever happened to actual behaviour? Perspectives on Psychological Science, 2, 4, 396403.

Belloc, N. and Breslow, L. (1972) The relationship of physical health status and health practices, Preventative Medicine, 1, 3, 409-21.

Harris, D. and Guten, S. (1979) Health-protective behavior: an exploratory study, Journal of Health and Social Behavior, 20, 1, 17-29.

Kasl, S. and Cobb, S. (1966) Health behavior, illness behavior and sick role behavior, Archives of Environmental Health, 12, 2, 246-66.

Leventhal, H., Brisette, I. and Leventhal, E. (2003) The common-sense model of self-regulation of health and illness. In Cameron, L.D. and Leventhal, H. (eds) The Self-regulation of Health and Illness Behavior. London: Routledge.

Matarazzo, J.D. (1983) Behavioral immunogens and pathogens: psychology's newest challenge, Professional Psychology: Research and Practice, 14, 3, 414-16.

Thaler, R. and Sustein, C. (2008) Nudge: Improving Decisions About Health, Wealth and Happiness. New Haven: Yale University Press.

Watson, J. (1930) Behaviorism. Chicago: University of Chicago Press. 